\title{
In vivo characterization of CYP2D6*12, *29 and *84 using dextromethorphan as a probe drug: a case report
}

\begin{abstract}
CYP2D $6 * 84$ was first described in a Black South African subject, however, its function remains unknown. Astrolabe, a probabilistic scoring tool developed in our laboratory to call genotypes from whole genome sequence, identified CYP2D6*84 in a trio. The father presented with intermediate metabolism when challenged with the CYP2D6 probe drug dextromethorphan $(D M /$ dextrorphan $[D X]=0.0839)$. Since his second allele, CYP2D $6 * 12$, is nonfunctional, the observed activity is derived by CYP2D $6 * 84$. This finding suggests that the allele's hallmark P267H causes decreased activity toward $\mathrm{DM}$ and that this allele should receive a value of 0.5 for Activity Score calculations. The mother's DM/DX of 0.0543 was consistent with the decreased activity classification of CYP2D6*29. The child, a critically ill neonate, was not phenotyped, but predicted to be a normal metabolizer.
\end{abstract}

First draft submitted: 8 December 2016; Accepted for publication: 13 January 2017; Published online: 14 March 2017

Keywords: $C Y P 2 D 6 \bullet C Y P 2 D 6 * 12 \bullet C Y P 2 D 6 * 84 \bullet$ dextromethorphan $\bullet$ phenotyping

Although CYP2D6 is among the best characterized drug-metabolizing enzymes, there is no information regarding function for many of the allelic variants described by the Human Cytochrome P450 (CYP) Allele Nomenclature Database [1]. Indeed, of the 109 defined allelic variants, function for 47 variants remains unknown or has only been investigated in vitro per the recent update of the Clinical Pharmacogenetics Implementation Consortium (CPIC) guideline for CYP2D6 and CYP2C19 genotypes and dosing of tricyclic antidepressants [2]. This information void can be attributed to a number of factors including the use of deidentified subjects in the detection of novel variants through sequencing studies making subjects unavailable for follow-up phenotyping, and the relative low frequency of those variants making it difficult to not only identify individuals carrying the variant of interest but also to identify subjects with an informative diplotype. As next-generation sequencing (NGS) platforms are increasingly employed for gene analysis in both the research and clinical settings, the detection of rare variants, including many of the 47 alleles currently classified as unknown function by CPIC, will become routine. Thus, the absence of information regarding the activity of these alleles will limit the translation of patient diplotypes into predicted phenotypes [2-5].

The $C Y P 2 D 6^{*} 84$ allele was first described in 2013 by Dodgen et al. in a Black South African subject by sequence analysis [6], but no additional information regarding this variant has emerged in the literature since its discovery. We have previously identified $C Y P 2 D 6^{*} 84$ in one subject of a trio (subject Children's Mercy Hospital [CMH] 631 [7]) using a bioinformatic tool, Astrolabe (initially named 'Constellation' $[7,8]$ ) that facilitates CYP2D6 genotype calling from whole genome sequence data. Astrolabe has now identified this allele in two additional sub-
Andrea Gaedigk ${ }^{*, 1,2}$, Greyson P Twist ${ }^{3}$, Emily G Farrow ${ }^{2,3}$, Jennifer A Lowry ${ }^{1,2}$, Sarah E Soden ${ }^{2,3}$ \& Neil A Miller ${ }^{3}$ 'Division of Clinical Pharmacology, Toxicology \& Therapeutic Innovation, Children's Mercy Kansas City, Kansas City, MO 64108, USA

${ }^{2} \mathrm{~S} c h o o l$ of Medicine, University of Missouri-Kansas City, Kansas City, MO 64108, USA

${ }^{3}$ Center for Pediatric Genomic Medicine, Children's Mercy Kansas City, Kansas City, MO 64108, USA *Author for correspondence:

Tel.: +1 8162343059

Fax: +1 8168551958 agaedigk@cmh.edu
Future
Medicine
part of 
jects of another trio, a father and a critically ill neonate. However, due to the unknown functional status of $C Y P 2 D 6^{*} 84$, we were unable to predict the phenotype of the individuals carrying this allele with a priori data.

This case report describes confirmatory Sanger resequencing of the alleles found in the second trio including $C Y P 2 D 6^{*} 84$. Both parents have genotypes consisting of known nonfunctional alleles that allowed us to determine the activity of $C Y P 2 D 6^{*} 84$ and confirm activity of $C Y P 2 D 6^{*} 29$ in these individuals with the CYP2D6 probe drug dextromethorphan (DM). The urinary metabolic ratio of DM over its metabolite dextrorphan (DX) served as a measure of CYP2D6 activity and thereby determines allele function and phenotype status [9].

\section{Methods}

This study was approved by the Institutional Review Board of Children's Mercy Kansas City. Informed written consent was obtained from the parents of the trios.

Whole genome sequence was performed as previously described [7,10]. Briefly, a library was constructed with the TruSeq DNA PCR-Free Library Prep Kit (Illumina, San Diego, CA, USA) according to the manufacturer's protocol and sequenced on an Illumina HiSeq 2500 instrument using paired end 100-bp reads to a read depth of at least $30 \times$. Samples were aligned to the GRCh37.p13 reference genome with bwa-mem v.0.7.12 and variants called with the Genome Analysis ToolKit v.3.2-2 producing variants relative to the CYP2D6 ${ }^{*} 2$ reference [10]. Subsequently, CYP2D6 diplotypes were called using a version of Astrolabe that has been improved since it was first published [7]. Astrolabe is available through the Children's Mercy website [11]. Variants across the entire CYP2D6 gene locus were manually inspected to validate Astrolabe calls.

Allele-specific long-range PCR was performed as previously described [12]. Specifically, we amplified the CYP2D6 ${ }^{*} 84$ (child), *4, *29 (mother) and *12 (father) alleles and Sanger-sequenced the resulting XL-PCR fragments using BigDye Terminator chemistry and a 3730 DNA analyzer (Thermo Fisher Scientific, MA, USA).

For phenotyping, subjects self-administered $30 \mathrm{mg}$ of DM (Robitussin ${ }^{\circledR}$ cough syrup, NC, USA) after voiding their bladder. Urine was collected for $4 \mathrm{~h}$. DM and DX were measured by NMS Labs (PA, USA). The urinary metabolic ratio of DM/DX served as a measure of CYP2D6 activity. Classification into poor, intermediate, normal (formerly known as extensive [13]) and ultra-rapid metabolizer phenotype groups has previously been described. Specifically, intermediate metabolizers are defined as $0.03 \leq \mathrm{DM} / \mathrm{DX}<0.3$ [14].

The following databases were utilized to obtain information regarding allele frequencies: the $\mathrm{CMH}$ Variant Warehouse at the Center for Pediatric Genomic Medicine at Children's Mercy Kansas City [15], the SPHINX database, a resource of the eMERGE Network [16], the Exome Aggregation Consortium (ExAC) database [17] and the NHLBI Exome Sequencing Project Exome Variant Server (EVS) [18].

\section{Results \& discussion}

\section{Allelic variation}

Astrolabe called the following genotypes for the trio: CYP2D6* $4 x N /{ }^{*} 29$ (mother), CYP2D6* $12 /{ }^{*} 84$ (father) and ${ }^{*} 29 /{ }^{*} 84$ (child). Astrolabe calls were confirmed by Sanger sequencing of allele-specific $C Y P 2 D 6^{*} 4,{ }^{*} 12$, *29 and 84 XL-PCR fragments. CYP2D $6 * 4$ matched $100 \%$ with the $* 4 D$ sequence we have described previously (case 21, in Gaedigk et al. [19], data not shown). In contrast, as summarized in Supplementary Figure 1, CYP $2 D 6^{*} 12$, *29 and ${ }^{*} 84$ deviated from the definitions listed by the $\mathrm{P} 450$ nomenclature database.

The $C Y P 2 D 6^{*} 84$ allele matched $100 \%$ with the one we identified in our first trio (subject CMH 631 described by Twist et al. [7]), but lacks two SNPs, one in intron 7 and one in the upstream region compared with the variant described by Dodgen et al. [6] on which the nomenclature is based for this allele. Interestingly, the $C Y P 2 D 6^{*} 84$ recently described by Chaudhry et al. [20], which matches $C Y P 2 D 6^{*} 84$ var, was found in a subject recruited from the same geographical region as the individual in which this variant was first discovered [6]. The $C Y P 2 D 6^{*} 12$ allele was first described in 1996 and at that time defined by variations in exon regions only, in other words, it is unknown whether there are SNPs in introns and/or the $3^{\prime}$ - and 5'-UTRs. Although these SNPs do not contribute to the function of this particular allele, having precise haplotype definitions facilitates automated diplotype calling using bioinformatic tools such as Astrolabe [7]. As shown in Supplementary Figure 1, the CYP $2 D 6^{*} 12$ in our trio features the 'key' SNP defining this allele $(124 \mathrm{G}>\mathrm{A}, \mathrm{G} 42 \mathrm{R}$, rs5030862), but also carries three additional exonic SNPs (1716G $>$ A, E155K, rs28371710; 2575C $>A$, rs 28371718 and $3254 \mathrm{~T}>\mathrm{C}$, rs28371726). However, the latter are not novel per se, as these SNPs are part of the CYP2D6*45 and * 46 haplotype definitions. A search of the CMH Variant Warehouse for the CYP2D6*12 key SNP (rs5030862) identified a second subject besides the father of the trio investigated here. Furthermore, this subject also has the three additional SNPs $(1716 \mathrm{G}>\mathrm{A}, 2575 \mathrm{C}>\mathrm{A}$ and $3254 \mathrm{~T}>\mathrm{C}$ ) suggesting that this $C Y P 2 D 6^{*} 12$ allele is 
Table 1. Allele frequencies of rs148382141, the key SNP defining CYP2D6*84.

\begin{tabular}{|c|c|c|c|c|}
\hline Database & Subjects (n) & $\begin{array}{l}\text { MAF (\%) all } \\
\text { subjects }\end{array}$ & $\begin{array}{l}\text { MAF (\%) African } \\
\text { ancestry }\end{array}$ & $\begin{array}{l}\text { MAF (\%) } \\
\text { European } \\
\text { ancestry }\end{array}$ \\
\hline $\mathrm{CMH}$ variant warehouse & 3432 & 0.044 & NA & NA \\
\hline SPHINX database & 9015 & 0.00555 & 0.0072 & 0 \\
\hline ExAC & 21,521 & 0.02567 & 0.2759 & 0 \\
\hline EVS & 6478 & 0.01003 & 0.2964 & 0 \\
\hline \multicolumn{5}{|c|}{$\begin{array}{l}\text { SPHINX data as per update of July 2015; CMH variant warehouse and ExAC accessed } 22 \text { November 2016. The CYP2D6*84-defining SNP } \\
\text { rs148769737 (Chr22:42524219 T/A) was not observed in the ExAC database among south or east Asians subjects but was discovered in } \\
\text { Latino and Europeans (non-Finnish) subjects at frequencies of } 0.0000978 \text { and } 0.00003349 \text {, respectively. } \\
\text { CMH: Children's Mercy Hospital; EVS: Exome variant server; ExAC: Exome Aggregation Consortium; MAF: Minor allele frequency; NA: No } \\
\text { available. }\end{array}$} \\
\hline
\end{tabular}

identical to the subvariant we have Sanger-sequenced for this study. Considering the presence of these additional SNPs of which one is nonsynonymous, the CYP2D6* 12 identified in this study in two subjects is more accurately described as a subvariant.

$C Y P 2 D 6^{*} 29$ is another allele for which the nomenclature database only lists variations in exons. The mother's CYP2D6*29 allele carried all exonic SNPs listed by the database, but also had numerous SNPs across introns and untranslated regions (Supplementary Figure 1). This $C Y P 2 D 6^{*} 29$ sequence matched those obtained from two Coriell samples, NA17131 and NA19109. However, this variant differed from a $C Y P 2 D 6^{*} 29$ sequence we obtained from a subject who participated in a previous study, but was not published in that report [21].

The P450 Nomenclature database does not assign star $\left({ }^{*}\right)$ allele designations for subvariants. Because there is currently no system in place that designates suballelic variants, the novel CYP2D6*12, *29 and *84 variants identified in this study are labeled with 'var'.

\section{Allele frequencies}

$C Y P 2 D 6^{*} 84$ is a rare variant that appears to be present predominantly in subjects with Black African ancestry (Table 1). The frequency of the key SNP identify- ing $C Y P 2 D 6^{*} 84(2574 \mathrm{C}>\mathrm{A}, \mathrm{P} 267 \mathrm{H}$, rs148769737) was $0.044 \%$ in the $\mathrm{CMH}$ Variant Warehouse (three heterozygous subjects among 3432). One of those subjects (CMH 631 described previously [7]) was genotyped as $C Y P 2 D 6^{*} 171^{*} 84$. Although the ethnicity of this subject is unknown, we speculate that this person is of African ancestry because of the presence of $C Y P 2 D 6^{*} 17$, which is almost exclusively found in African populations or populations with African admixture (see CYP2D6 allele frequency table available at the PharmGBK website $[22,23])$. The other two subjects are the father and child described in this report. Our Variant Warehouse frequency, albeit not derived from unrelated subjects only, is in line with those reported by the SPHINX and ExAC databases (Table 1). Taken together, these data suggest that $C Y P 2 D 6^{*} 84$ is predominantly present in African populations and subjects with African ancestry. The $C Y P 2 D 6^{*} 12$ allele also appears to be rare and, as shown in Table 2, has been found predominantly in subjects with African ancestry. However, two subjects with $C Y P 2 D 6^{*} 84$ were found in two studies conducted in South African subjects $(n=100[6]$ and $n=31$ [20], respectively), which suggest an allele frequency of $0.76 \%$, which is considerably higher compared with the frequencies reported by a number of databases (Table 1). Whether this allele

\section{Table 2. Allele frequencies of rs5030862, the key SNP defining CYP2D6*12.}

\begin{tabular}{|c|c|c|c|c|}
\hline Database & Subjects (n) & $\begin{array}{l}\text { MAF (\%) all } \\
\text { subjects }\end{array}$ & $\begin{array}{l}\text { MAF (\%) African } \\
\text { ancestry }\end{array}$ & $\begin{array}{l}\text { MAF (\%) } \\
\text { European } \\
\text { ancestry }\end{array}$ \\
\hline $\mathrm{CMH}$ variant warehouse & 3432 & 0.029 & NA & NA \\
\hline SPHINX database & 9015 & 0.0222074 & 0.148258 & 0 \\
\hline ExAC & 51,296 & 0.003899 & 0.03575 & 0.001748 \\
\hline EVS & 6491 & 0.0231 & 0.0455 & 0.0116 \\
\hline
\end{tabular}


is indeed more common in this particular population warrants further investigation.

\section{Phenotype prediction from genotype data} \& in vivo phenotyping using DM

Because of the 'unknown' function status of $C Y P 2 D 6^{*} 84$, the father's phenotype was predicted to be either a poor metabolizer (activity score $=0$ ), intermediate metabolizer (activity score $=0.5$ ) or normal metabolizer (activity score $=1$ ) depending on whether $C Y P 2 D 6^{*} 84$ is a no, decreased or normal function allele. The mother's genotype received an activity score of 0.5 predicting an intermediate metabolizer phenotype status based on the decreased function categorization of $C Y P 2 D 6^{*} 29$. The prediction was intermediate or normal metabolizer for the child (translation into phenotype according to the updated CPIC translation table [22]).

The father uniquely presented us with a rare opportunity to measure CYP2D6 activity in vivo that is solely derived from $C Y P 2 D 6^{*} 84$. Only about 1 in 10,000 individuals carries a genotype consisting of a nonfunctional allele that is paired with $C Y P 2 D 6^{*} 84$ such as the father's, in other words, $C Y P 2 D 6^{*} 121^{*} 84$. In vivo phenotyping determined a urinary metabolic ratio of $\mathrm{DM} / \mathrm{DX}$ of 0.0839 categorizing the father as intermediate metabolizer. The allele-defining SNP in exon 5 $(2574 \mathrm{C}>\mathrm{A})$ causes an amino acid change $(\mathrm{P} 267 \mathrm{H})$ that is predicted by SIFT to be damaging (score of 0.03) [24] and by PolyPhen to be probably damaging (score of 0.977 [25]. Decreased activity, as indicated by a urinary metabolic ratio of DM/DX of 0.0839 is consistent with the in silico predictions and might be explained by a difference in the charge caused by the proline to histidine change. Based on these findings, CYP2D6*84 should be classified as 'decreased' and receive a value of 0.5 for Activity Score calculations.

The mother's DM/DX ratio was 0.0543 classifying her as an intermediate metabolizer that was expected given her genotype $\left(C Y P 2 D 6^{*} 4 \times 2 /^{*} 29\right)$. Decreased activity is consistent with published data describing $C Y P 2 D 6^{*} 29$ as a variant encoding protein with compromised function $[21,26]$.
The child, a critically ill neonate was not subjected to phenotyping. Based on the parent's phenotype information, the child's diplotype $\left(C Y P 2 D 6^{*} 29 /{ }^{*} 84\right)$ was calculated to have an Activity Score of 1 predicting normal metabolism, which provided valuable information to parents and providers caring for this complex patient.

\section{Conclusion}

In vivo phenotyping of an individual with an informative CYP2D6 diplotype allowed us to determine $C Y P 2 D 6^{*} 84$ activity for the first time. This information is invaluable to predict CYP2D6 activity in patients carrying this allelic variant.

\section{Future perspective}

As genotype testing is increasingly performed using NGS platforms, rare alleles will reveal themselves, but will elude interpretation due to the lack of data. The development of protocols allowing follow-up phenotype studies are warranted to functionally characterize the increasing number of allelic variants and diplotype combinations discovered by NGS to accurately predict phenotype and aid thereby move individualized drug therapy forward.

\section{Supplementary data}

To view the supplementary data that accompany this paper, please visit the journal website at: www.futuremedicine.com/ doi/full/10.2217/pgs-2016-0192

\section{Financial \& competing interests disclosure}

The authors have no relevant affiliations or financial involvement with any organization or entity with a financial interest in or financial conflict with the subject matter or materials discussed in the manuscript. This includes employment, consultancies, honoraria, stock ownership or options, expert testimony, grants or patents received or pending, or royalties.

No writing assistance was utilized in the production of this manuscript.

\section{Executive summary}

- CYP2D6*84 is a rare variant predominantly observed in Africans and subjects with African ancestry. It was first discovered in a South African individual in 2013.

- Because CYP2D6*84 function remained unknown, phenotype could not be predicted, or only approximated, from diplotypes containing this allele.

- Astrolabe, a tool to call genotypes from next-generation sequencing data, correctly identified the CYP2D6*84 allele in three individuals of two trios (one trio described in this report).

- Allele calls were verified by Sanger sequencing and an individual with a CYP2D6*12/*84 genotype subjected to in vivo phenotyping using the CYP2D6 probe drug dextromethorphan.

- The urinary metabolic ratio of dextromethorphan to its metabolite dextrorphan (DM/DX) indicated that CYP2D6*84 encodes decreased function. This finding is consistent with in silico predictions. 


\section{Informed consent disclosure}

The authors state that they have obtained verbal and written informed consent from the patient/patients for the inclusion of their medical and treatment history within this case report.

\section{References}

1 The Human Cytochrome P450 (CYP) Allele Nomenclature Database. www.cypalleles.ki.se/

2 Kevin Hicks J, Sangkuhl K, Swen JJ et al. Clinical Pharmacogenetics Implementation Consortium guideline (CPIC [R]) for CYP2D6 and CYP2C19 genotypes and dosing of tricyclic antidepressants: 2016 update. Clin. Pharmacol. Ther. doi:10.1002/cpt.597 (2016) (Epub ahead of print).

3 Crews KR, Gaedigk A, Dunnenberger HM et al. Clinical Pharmacogenetics Implementation Consortium (CPIC) guidelines for cytochrome P450 2D6 (CYP2D6) genotype and codeine therapy: 2014 update. Clin. Pharmacol. Ther. 95(4), 376-382 (2014).

4 Bell GC, Caudle KE, Whirl-Carrillo M et al. Clinical Pharmacogenetics Implementation Consortium (CPIC) guideline for CYP2D6 genotype and use of ondansetron and tropisetron. Clin. Pharmacol. Ther. doi:10.1002/cpt.598 (2016) (Epub ahead of print).

5 Hicks JK, Bishop JR, Sangkuhl K et al. Clinical Pharmacogenetics Implementation Consortium (CPIC) guideline for CYP2D6 and CYP2C19 genotypes and dosing of selective serotonin reuptake inhibitors. Clin. Pharmacol. Ther. 98(2), 127-134 (2015).

6 Dodgen TM, Hochfeld WE, Fickl H et al. Introduction of the AmpliChip CYP450 test to a South African cohort: a platform comparative prospective cohort study. BMC Med. Genet. 14, 20 (2013).

7 Twist G, Gaedigk A, Miller N et al. Constellation: a tool for rapid, automated phenotype assignment of a highly polymorphic pharmacogene, CYP2D6, from whole-genome sequence. Genomic Med. doi:10.1038/npjgenmed.2015.7 (2016) (Epub ahead of print).

8 Twist GP, Gaedigk A, Miller NA et al. Constellation: a tool for rapid, automated phenotype assignment of a highly polymorphic pharmacogene, CYP2D6, from whole-genome sequences. Corrigendum. Npj Genomic Med. 2, 16039 (2017).

9 Frank D, Jaehde U, Fuhr U. Evaluation of probe drugs and pharmacokinetic metrics for $C Y P 2 D 6$ phenotyping. Eur. J. Clin. Pharmacol. 63(4), 321-333 (2007).

10 Saunders CJ, Miller NA, Soden SE et al. Rapid wholegenome sequencing for genetic disease diagnosis in neonatal intensive care units. Sci. Transl. Med. 4(154), 154ra135 (2012).

11 Children's Mercy Center for Pediatric Genomic Medicine. www.childrensmercy.org/genomesoftwareportal/

12 Gaedigk A, Riffel AK, Leeder JS. CYP2D6 haplotype determination using long range allele-specific amplification:

\section{Open access}

This work is licensed under the Creative Commons Attribution-NonCommercial 4.0 Unported License. To view a copy of this license, visit http://creativecommons.org/licenses/bync-nd/4.0/

resolution of a complex genotype and a discordant genotype involving the CYP2D6*59 allele. J. Mol. Diagn. 17(6), 740-748 (2015).

13 Caudle KE, Dunnenberger HM, Freimuth RR et al. Standardizing terms for clinical pharmacogenetic test results: consensus terms from the Clinical Pharmacogenetics Implementation Consortium (CPIC). Genet. Med. 19(2), 215-223 (2016).

14 Gaedigk A, Simon SD, Pearce RE, Bradford LD, Kennedy MJ, Leeder JS. The CYP2D6 activity score: translating genotype information into a qualitative measure of phenotype. Clin. Pharmacol. Ther. 83(2), 234-242 (2008).

15 CMH Variant Warehouse. http://warehouse.cmh.edu

16 SPHINX. www.emergesphinx.org

17 ExAC Browser. Exome Aggregation Consortium. http://exac.broadinstitute.org

18 NHLBI Exome Sequencing Project (ESP). Exome Variant Server.

http://evs.gs.washington.edu/

19 Gaedigk A, Freeman N, Hartshorne T et al. SNP genotyping using TaqMan technology: the $C Y P 2 D 6^{*} 17$ assay conundrum. Sci. Rep. 5, 9257 (2015).

20 Chaudhry M, Alessandrini M, Rademan J et al. Impact of CYP2D6 genotype on amitriptyline efficacy for the treatment of diabetic peripheral neuropathy: a pilot investigation. Pharmacogenomics In Publication, (2017).

21 Gaedigk A, Bradford LD, Marcucci KA, Leeder JS. Unique CYP2D6 activity distribution and genotype-phenotype discordance in black Americans. Clin. Pharmacol. Ther. 72(1), 76-89 (2002).

22 PharmGKB. www.pharmgkb.org/page/cyp2d6RefMaterials

23 Gaedigk A, Sangkuhl K, Whirl-Carrillo M, Klein T, Leeder JS. Prediction of CYP2D6 phenotype from genotype across world populations. Genet. Med. 19(1), 69-6 (2017).

24 Kumar P, Henikoff S, Ng PC. Predicting the effects of coding non-synonymous variants on protein function using the SIFT algorithm. Nat. Protoc. 4(7), 1073-1081 (2009).

25 Adzhubei IA, Schmidt S, Peshkin L, et al. A method and server for predicting damaging missense mutations. Nat. Methods 7(4), 248-249 (2010).

26 Wennerholm A, Johansson I, Hidestrand M, Bertilsson L, Gustafsson LL, Ingelman-Sundberg M. Characterization of the $C Y P 2 D 6^{*} 29$ allele commonly present in a black Tanzanian population causing reduced catalytic activity. Pharmacogenetics 11, 417-427 (2001). 